J. Clin. Chem. Clin. Biochem.

Vol. 17,1979 , pp. 523-527

\title{
Geschlechtsunterschiede der Triglycerid- und Cholesterinzusammensetzung des „,normalen“ Serumlipoproteinmusters (sog. UZ-Lipidstatus)
}

\author{
Von K. H. Vogelberg und N. Szymanski \\ Klinische Abteilung des Diabetes-Forschungsinstitutes an der Universität Düsseldorf
}

(Eingegangen am 26. Oktober 1978/19. Februar 1979)

\begin{abstract}
Zusammenfassung: Die bei Männern und Frauen unterschiedliche Arterioskleroseneigung ist wiederholt mit der verschiedenen Triglycerid- und Cholesterinkonzentration der Serumlipoproteine in Zusammenhang gebracht worden. Die Ursache für den Geschlechtsunterschied ist unbekannt. Bei 100 klinisch gesunden Personen, je 50 Männern (im Mittel 30 Jahre, relatives Körpergewicht nach Broca: 0,942) und 50 Frauen (im Mittel 31 Jahre, relatives Körpergewicht nach Broca: 0,971) wurden die Lipide bestimmt und die Bedeutung geschlechtsabhängiger Konzentrationsunterschiede untersucht.
\end{abstract}

Die Untersuchung ergab, daß bei Männern die HDL-Cholesterinkonzentration kleiner war als bei Frauen $(1,13$ vs. $1,49 \mathrm{mmol} / \mathrm{l})$. Außerdem war festzustellen, daß bei Männern im Durchschnitt VLDL-Triglyceridkonzentrationen oberhalb des Medians größer waren als bei Frauen (0,91 vs. 0,68 mmol/1). Eine direkte Beeinflussung des HDLCholesterins durch VLDL-Triglyceride war in Vergleichsgruppen nicht nachweisbar. Bei multipler Regressionsanalyse zeigte sich jedoch, daß bei Männern ein indirekter Einfluß durch VLDL-Triglyceride nicht auszuschließen war. Die Variabilität des HDL-Cholesterins wurde bei Männern fast 10 mal stärker durch VLDL-Triglyceride bestimmt als bei Frauen. Es wird angenommen, daß die bei Männern niedrigere HDL-Cholesterinkonzentration z.T. von der aktuellen VLDL-Triglyceridkonzentration abhängig ist.

Sex differences in the triglyceride and cholesterol composition of the fractions constituting the "normal" pattern of serum-lipoproteins (so-called ultracentrifugal lipid status)

Summary: A connection has again been demonstrated between the different tendency of men and women to arteriosclerosis and the differences in the concentrations of triglycerides and cholesterol in the serum lipoproteins. The reason for this sex difference is unknown. Lipids were determined in 50 clinically healthy men (average age 30 years, relative body weight according to Broca, 0.942) and in 50 clinically healthy women (average age 31 years, relative body weight according to Broca, 0.971 ), and possible interpretations of the sex-dependent concentration differences were investigated.

The HDL cholesterol concentration was lower in men than in women (1.13 and $1.49 \mathrm{mmol} / \mathrm{l})$. It was also found that VLDL triglyceride concentrations above the median were on average higher in men than in women $(0,91$ and 0.68 $\mathrm{mmol} / \mathrm{l})$. A direct effect of VLDL triglycerides on the HDL cholesterol could not be demonstrated. Multiple regression analysis showed, however, that an indirect effect of VLDL triglycerides could not be excluded. Correlation between the variability of the HDL cholesterol and the level of VLDL triglycerides was ten times greater in men than in women. It is suggested that in men, the lower HDL cholesterol concentration is partly dependent on the circulating concentration of VLDL triglycerides.

\section{Einführung}

Die Bestimmung der Triglycerid- und Cholesterinzusammensetzung der Serumlipoproteine (sog. UZ-Lipidstatus) hat für die diagnostische Einteilung („Typisierung ") verschiedener Fettstoffwechselstörungen grọe Bedeutung erlangt (1).
Die Methode hat wesentlich dazu beigetragen, unsere Kenntnisse über spezifische Funktionen einzelner Lipoproteine im Zusammenhang mit der Arteriosklerose zu erweitern. In den letzten Jahren ist diesbezüglich vor allem die bei Männern im Vergleich zu Frauen geringere HDLCholesterinkonzentration herausgestellt wọrden. Die Ur- 
sache für diesen Geschlechtsunterschied ist unbekannt. Da coronare Gefäßkrankheiten durch niedrige HDLCholesterinkonzentrationen wahrscheinlich begünstigt werden (2), ist dieser Geschlechtsunterschied jedoch pathogenetisch besonders wichtig.

Nachfolgend wurde bei klinisch gesunden Personen (je 50 Männer und 50 Frauen) ohne klinisch nachweisbare Arteriosklerosezeichen der UZ-Lipidstatus bestimmt und die Bedeutung von Konzentrationsunterschieden zwischen den Geschlechtern untersucht.

\section{Personenauswahl}

Bei den untersuchten Personen handelte es sich um normgewichtige Angestellte (Ärzte, Pflege- und Laborpersonal) des Diabetes-Forschungsinstituts an der Universität Düsseldorf, bei denen eine primäre oder sekundäre Stoffwechselstörung infolge einer Leber-, Pankreas-, Nieren- oder endokrinen Erkrankung ausgeschlossen worden war. Kriterien hierfür waren sowohl anamnestische als auch laborchemische Befunde (GlutamatOxalacetat-Transaminase, Amylase, Creatinin und Thyroxin bzw. Trijodthyronin im Serum). Den Stoffivechsel beeinflussende Medikamente wurden nicht eingenommen und ein chronischer Alkoholkonsum war nicht bekannt. EKG-Befunde (Extremitäten-, Brustwand- und Nehb-Ableitungen) und peripherer Gefäßstatus (Gefäßpulse) waren unauffällig. Das Alter der Personen schwankte zwischen 19 und 49 Jahren und betrug im Mittel (Median) $31 \mathrm{Jahre}$. Das relative Körpergewicht wurde bei leichter Körperbekleidung (ohne Schuhe, Kittel und/oder Jacke) bestimmt und als Fraktion des Sollgewichts nach Broca definiert, wobei Frauen 0,10 vom Sollgewicht abgezogen wurde. Personen mit einem relativen Broca-Gewicht über 1,10 bzw. unter 0,80 wurden von der Untersuchung ausgeschlossen.

\section{Methoden}

Sämtliche Lipidanalysen wurden aus Blutproben durchgeführt, die morgens nüchtern aus einer ungestauten Armvene entnommen worden waren. Die Seren wurden bei Raumtemperatur abzentrifugiert $(2500 \mathrm{U} / \mathrm{min})$ und bis zum Zeitpunkt der Untersuchung auf Eis konserviert. Die Triglycerid-, Cholesterin- und Glucosebestimmung im Serum erfolgte enzymatisch mit Hilfe 'herkömmlicher Methoden (3,4,5). Die Fraktionierung der Lipoproteine erfolgte mit einer Spinco L2-65 Ultrazentrifuge der Firma Beckman. Je $4 \mathrm{ml}$ Serum wurden in $2 \mathrm{ml}$ phy siologischer $\mathrm{NaCl}-L o ̈ s u n g$ verdünnt und in einem 40.3-Rotor bei $105000 \mathrm{~g}$ über 20 Stunden bei $5^{\circ} \mathrm{C}$ zentrifugiert. VLDL wurden bei einer Dichte von d $=1,006$ mit Hilfe eines "tube sclicer" von LDL und HDL abgetrennt und LDL mit Heparin und Manganchlorid präzipiert (6). Der LDL-Cholesteringehalt im Unterstand und der die HDL enthaltenden Lösung wurden nach Polyanionenpräzipitation ermittelt (7). Pro Serum wurden 2 Proben ultrazentrifugiert und der Lipidgehalt im Nativserum bzw. in einzelnen Serumlipoproteinfraktionen je 3 fach bestimmt. Der mittlere Variationskoeffizient der ind ividuellen Bestimmung war für Cholesterin 1,7 $\pm 1,2 \%$ und für Triglyceride $2,4 \pm 1,8 \%$. Der Konzentrationsverlust im Verlauf der Ultrazentrifugation einzelner Lipoproteinfraktionen lag im Durchschnitt aller Triglycerid- und Cholesterinbestimmungen bei $8,5 \pm 2,1 \%$.

\section{Statistik ${ }^{\mathbf{1}}$ )}

Sämtliche Untersuchungsergebnisse wurden als Mittelwerte mit einfacher Standardabweichung angegeben. Aufgrund des großen Variationskoeffizienten der Einzelwerte (über 33\%) wurde für die Berechnung der Mittelwerte eine log-normale Verteilung angenommen. Auf statistische Signifikanzberechnungen wurde ver-

1) Für statistische Beratung danken wir Herrn Dipl. Volkswirt $K$. Dannehl, Abteilung für Med. Statistik und Epidemiologie des Diabetes-Forschungsinstituts an der Universität Düsseldorf. zichtet, da es sich bei den untersuchten Personen nicht um eine zufällige Auswahl von Probanden aus einer größeren Zielpopulation handelte. Die untersuchten Männer und Frauen wurden vielmehr, wie enwähnt, anhand von Einschluß-bzw. Ausschlußkriterien ausgewählt. Auf einfache Korrelationsberechnungen wurde ebenfalls verzichtet; stattdessen wurde mit Rücksicht auf die Vielzahl verschiedener Einflußmöglichkeiten auf bestimmte Lipidkenngrößen eine multiple Regressionsanalyse vorgenommen. Das willkürlich additive (lineare) Modell dieser Bcrechnung wurde inkaufgenommen.

\section{Ergebnisse}

Tabelle 1 zeigt, daß das relative Körpergewicht bei Frauen etwa $1 / 30$ größer war als bei Männern; die mit tlere Triglycerid- und Cholesterinkonzentrationen in Nativserum und UZ-Lipidstatus wichen jedoch bei beiden Geschlechtern nur wenig voneinander ab (Tab. 2). Die Triglyceridkonzentration war bei Männern im Nativserum, in VLDL und LDL zwar größer als bei Frạuen, ein deutlicher Unterschied war aber lediglich zwischen der HDLCholesterinkonżentration nachweisbar: die Konzentration war bei Männern etwa $1 / 3$ kleiner als bei Frauen $(1,13$ vs. $1,49 \mathrm{mmol} / \mathrm{l})$.

Die kumulative Häufigkeitsverteilung bestimmter Konzentrationen ist in Abbildung 1 dargestellt. Der Kurvenverlauf żeigt, daß lediglich für LDL- und HDL-Chole-

Tab. 1. Anzahl, Alter, relatives Körpergewicht und mittlere Blutglucosekonzentration von 100 stoff wechselgesunden Personen.

\begin{tabular}{lll}
\hline & $\circ$ & 9 \\
\hline Anzahl & 50 & 50 \\
Alter (Jahre) & $30(19-49)$ & $31^{*}(19-49)$ \\
$\begin{array}{l}\text { Rel. Körpergewicht } \\
\text { (Broca) }\end{array}$ & $0,942 \pm 0,080$ & $0,971 \pm 0,072^{* *}$ \\
Blutglucose (mmol/l) & $84,1(74,5 ; 95,1)$ & $86,3(77,9 ; 95,5)^{* * *}$ \\
\hline
\end{tabular}

*) Median, Minimum und Maximumangabe in Klammern

**) Arithmetrischer Mittelwert \pm Standardabweichung

***) Geometrischer Mittelwert mit einfacher Standardabweichung in Klammern

Tab. 2. Geometrische Mittelwerte der Triglycerid- und Cholesterinkonzentration in Nativserum und UZ L-Lipidstatus (angegeben in mmol/l Serum mit einfacher Standardabweichung in Klammern) bei je 50 stoffwechselgesunden Männern und Frauen.

\begin{tabular}{|c|c|c|}
\hline & 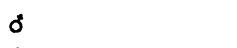 & 8 \\
\hline Triglyceride & $0,89(0,55 ; 1.42)$ & $0,79(0,52 ; 1,20)$ \\
\hline Cholesterin & $4,63(3,57 ; 6,00)$ & $4,81(3,78 ; 6,13)$ \\
\hline Triglyceride & $0,51(0,27 ; 0,97)$ & $0,42(0,24 ; 0,74)$ \\
\hline Cholesterin & $0,21(0,07 ; 0 ; 67)$ & $0,20(0,08 ; 0,51)$ \\
\hline Triglyceride & $0,15(0,05 ; 0,43)$ & $0,15(0,06 ; 0,39)$ \\
\hline Cholesterin & $2,99(2,02 ; 4,44)$ & $2,92(2,12 ; 4,02)$ \\
\hline Trigly ceride & $0,11(0,04 ; 0,28)$ & $0,09(0,02 ; 0,31)$ \\
\hline Cholesterin & $1,13(0,71 ; 1,78)$ & $1,49(1,03 ; 2,15)$ \\
\hline
\end{tabular}



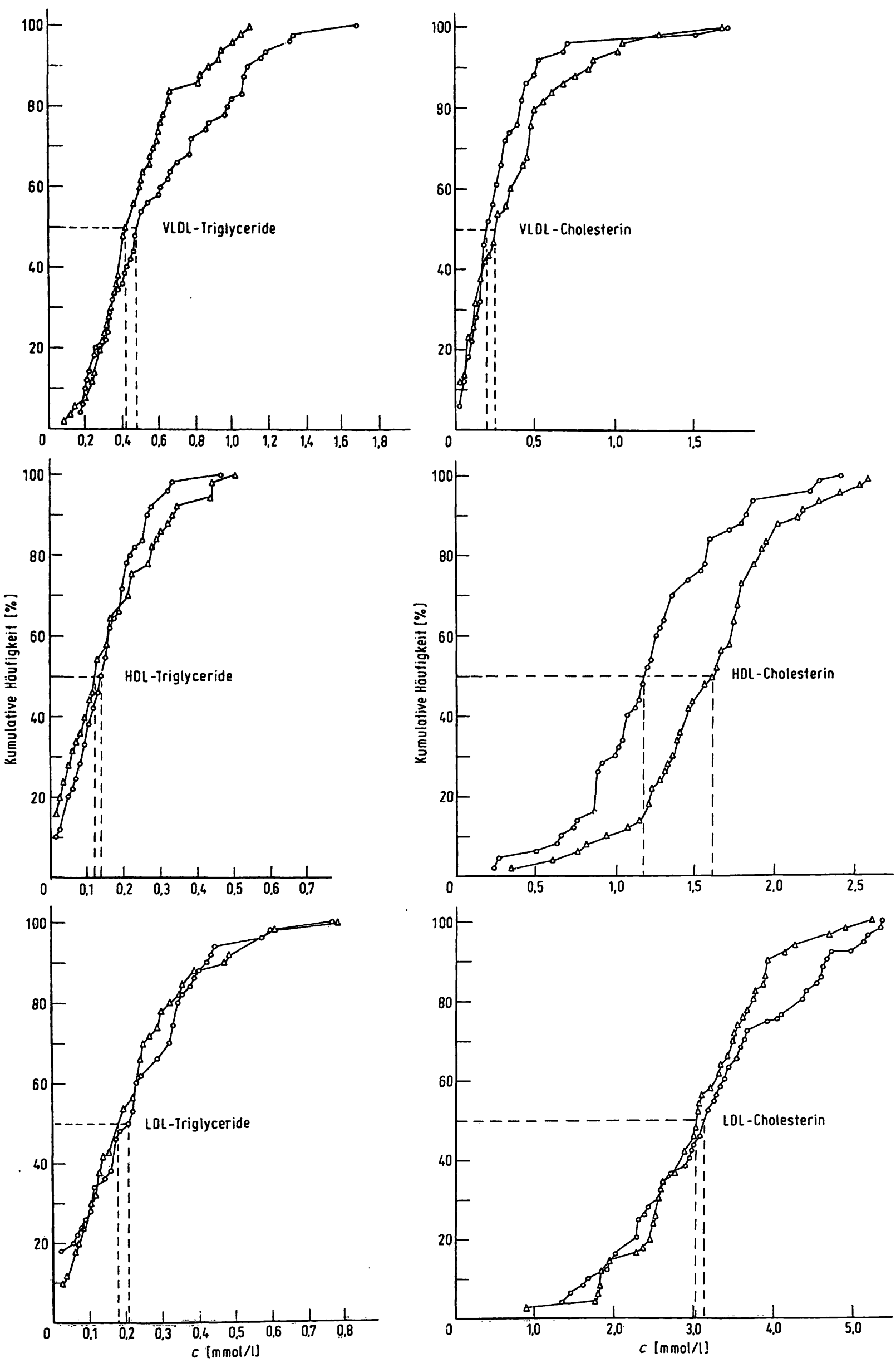

Abb. 1. Kumulative Häufigkeitsverteilung (angegeben in \%) einzelner Triglycerid- und Cholesterinwerte des UZ-Lipid status (angegeben in mmol/1 Serum) bei je 50 stoff wechselgesunden Männern $(0-0)$ und Frauen $(\Delta-\Delta)$ (Median gestrichelt). 
sterin eine normale Verteilung hätte angenommen werden können, und dementsprechend der arithmetrische Mittelwert besser mit dem Median übereinstimmte (Tab. 2). Die Abbildung zeigt außerdem, daß nicht nur für HDL-Cholesterin, sondern auch für VLDL-Triglyceride zwischen beiden Geschlechtern deutliche Unterschiede bestanden. Während bei HDL-Cholesterin bereits die durchschnittliche Konzentration aller Einzelwerte voneinander abwich, traf dies für VLDL-Triglyceride jedoch nur für die durchschnittliche Konzentration einzelner Werte oberhalb des Medians zu: die VLDL-Triglyceridkonzentration war bei Männern etwa $1 / 3$ größer als bei Frauen $(0,91$ vs. $0,68 \mathrm{mmol} / \mathrm{l})$.

Der Geschlechtsunterschied von HDL-Cholesterin ist anhand von Vergleichsgruppen und mittels multipler Regression analysiert worden. Tabelle 3 zeigt, daß der Geschlechtsunterschied auch dann erkennbar war, wenn er bei Männern und Frauen mit gleichem VLDL-Triglycerid-bzw. LDL-Cholesterin untersucht wurde. Ein direkter Zusammenhang mit diesen Lipiden war dementsprechend nicht nachweisbar. Im Gesamtspektrum aller untersuchten Variablen konnte jedoch ein Einfluß des VLDL-Triglycerid nicht ausgeschlossen werrden. Das Ergebnis der multiplen Regression zeigte, daß die Variabilität des HDL-Cholesterins bei Männern fast 10 mal stärker durch VLDL-Triglyceride bestimmt wurde und im Unterschied zu Frauen auch ein indirekter $\mathrm{Zu}$ sammenhang zu VLDL-Cholesterin erkennbar war.

Tab. 3. HDL-Cholesterinkonzentration bei Personen mit gleicher VLDL-Triglyceridkonzentration, angegeben als geometrische Mittelwerte in mmol/1 Serum mit einfacher Standardabweichung in Klammern. Für die Durchführung der Stichproben wurde die jeweils größtmögliche Fallzahl von Einzelbefunden ausgewählt.

\begin{tabular}{lll}
\hline & $\delta$ & 9 \\
\hline Anzahl & 31 & 31 \\
VLDL-Triglyceride & $0,52(0,28 ; 0,75)$ & $0,52(0,25 ; 0,79)$ \\
HDL-Cholesterin & $1,25(0,91 ; 1,69)$ & $1,49(1,03 ; 1,84)$ \\
\hline
\end{tabular}

Tab. 4. Bestimmtheitsmaße der multiplen Regression der HDLCholesterinkonzentration durch Alter, relatives Körpergewicht (Broca) Blutglucose-, und Lipidkonzentration in Nativserum und UZ-Lipidstatus (angegeben in \%).

\begin{tabular}{|c|c|c|c|}
\hline & 8 & \$ & \\
\hline Alter & 0,04 & 0,06 & \\
\hline Körpergewicht (Broca) & 1,63 & 2,21 & \\
\hline \multirow{2}{*}{$\begin{array}{l}\text { Blutglucose } \\
\text { Triglyceride }\end{array}$} & 0,56 & 0,62 & $\cdots$ \\
\hline & 15,31 & 1,61 & \\
\hline Cholesterin & 14,69 & 0,12 & \\
\hline Triglyceride & 4,11 & 1,78 & \\
\hline Cholesterin & 0,07 & 0,19 & \\
\hline HDL-Triglyceride & 0 & 2,26 & \\
\hline Total & 36,41 & 8,85 & \\
\hline
\end{tabular}

\section{Diskussion}

Die vorliegenden Untersuchungen sind aufgrund der aufwendigen Auswahl klinisch gesunder Personen nur an einer relativ kleinen Anzahl von Personen durchgeführt worden. Die Ergebnisse können nicht für Normalpersonen der Durchschnittsbevölkerung repräsentätiv sein, sondern lediglich als Normalbefunde bei Personen innerhalb einer Hochschulinstitution angesehẻn werden. Das Lebensalter wurde auf 19-49 Jahre begrenzt, um Geschlechtsunterschiede möglichst gut erkennen zu können.

Der geschlechtsabhängige Unterschied des HDL-Cholesterins ist bereits seit langem bekannt (8). Die Mittelwerte stimmen gut mit früheren Untersuchungen überein: Havel et al (9) fanden bei 20-30-jährigen stoffwechselgesunden Personen - es handelte sich ebenfạlls um Ärzte, Pिflege- und Laborpersonal - einen Mittelwert von $43 \pm 5$ bei Männern und $45 \pm 11 \mathrm{mg} / \mathrm{dl}^{2}$ ) Serum bei Frauen. Carlson \& Ericsson (10) ermittelten bei 30- bis 39-jährigen Stadtbewohnern Uppsalas einen entsprechenden Wert von $45 \pm 3$ bzw. $64 \pm 3 \mathrm{mg} / \mathrm{dl}$. Mj $\phi s$ et al (11) bestimmten bei 30-39-jährigen Familienmitgliedern der Troms $\phi$-Studie eine Konzentration von $44,2 \pm 12$ bzw. $51,6 \pm 12,1 \mathrm{mg} / \mathrm{dl}$ Serum. Eine Zusammenstellung zahlreicher epidemiologischer Studien aus verschiedenen Bevölkerungsgruppen in den USA (12) zeigte, daß bei Männern im Alter zwischen 40 und $49 \mathrm{~J}$ ahren auch trotz rassischer, geographischer oder anderer Unterschiede stets eine niedrigere HDL-Cholesterinkonzentration als bei gleichaltrigen Frauen vorliegt.

Bei Untersuchungen an 35 bis 39-jährigen Langstreckenläufern war HDL-Cholesterin mit $59 \pm 10 \mathrm{mg} / \mathrm{dl}$ Serum erhöht (13). In einem Kontrollkollektiv von Herzinfarktpatienten (Durchschnittsalter $44 \mathrm{~J}$ ahre) betrug die Konzentration bei Männern ebenfalls lediglich $45 \pm 11,8$ $\mathrm{mg} / \mathrm{dl}$ Serum (14).

In verschiedenen, namentlich epidemiölogischen Untersuchungen ist die Abhängigkeit des HDL-Cholesterins von verschiedenen Faktoren. so auch vom Alter und Körpergewicht untersucht worden. Eine Beeinflussung durch das Alter war bisher nur innerhalb einer Familie, d.h. einem genetisch homogenen Personenkreis nachzuweisen (11). In der vorliegenden Arbeit war bei Frauen und Männern keine Altersabhängigkeit festzustellen. Sie bestätigt jedoch, daß die HDL-Cholesterinkonzentration entsprechend zahlreichen Voruntersuchungen $(15,16$, 17) gewichtsabhängig isț. Die Beziehung zum Gewicht könnte für die bei Männern niedrigere HDL-Cholesterinkonzentration grundsätzlich wichtig sein. Da das Körpergewicht der hier untersuchten Männer geringer war, könnte jedoch lediglich eine indirekte Beeinflussung angenommen werden.

Abgesehen vom Cholesterin ist bei Frauen auch die Phosphatidkonzentration und der $\alpha_{2}$-Apolipoproteingehalt größer als bei Männern $(9,18)$. Nach Untersuchungen

2) Zur Umrechnung von Cholesterin: $1 \mathrm{mg} / \mathrm{dl}$ Serum $=0,0259$ $\mathrm{mmol} / 1$ Serum 
von Anderson et al (19) beschränkt sich der Geschlechtsunterschied ausschließlich auf die HDL-2-Subfraktion. Albers et al (20) nehmen an, daß Östrogene für die höhere HDL-Cholesterinkonzentration verantwortlich sind. Die Bedeutung von Kontraceptiva für die HDLCholesterinkonzentration ist noch unklar. Während von Krauss et al (21) eine Steigerung der HDL-3-Subfraktion beobachtet wurde, ist von anderen Autoren eine Abnahme der Gesamt-HDL-Fraktion beschrieben worden $(22,23)$. Es ist wahrscheinlich, daß die gegensätzlichen Befunde durch eine unterschiedliche Östrogen-Progesteronzusammensetzung der Kontraceptiva hervorgerufen wird. Da der Geschlechtsunterschied der HDL-Cholesterinkonzentration bereits bei der Geburt vorhanden ist (24) und familiär eine X-chromosomale Vererbung vorliegt (25), können für den Unterschied nicht nur hormonelle Einflüsse verantwortlich gemacht werden.

Eine negative Beziehung der HDL-Cholesterinkonzentration zur VLDL- bzw. der Gesamttriglyceridkonzentration ist bei beiden Geschlechtern beschrieben worden $(15,16,17,26)$. Die vorliegenden Untersuchungen

\section{Literaturverzeichnis}

1. Fredrickson, D. S., Levy, R. I., Kwiterovich, P. P. \& Jover, A. (1969), in: Drugs Affecting Lipid-Metabolism (W. L. Holmes, L. A. Carlson \& R. Paoletti, eds.) Plenum Press, New York, S. 307.

2. Miller, G. J. \& Miller, N. E. (1975), Lancet $I, 16-19$.

3. Eggstein, M. \& Kreutz, F. M. (1966), Klin. Wochenschr. 44, $262-267$

4. Zlatkis, A., Zak, B. \& Boyle, A. J. (1959), J. Lab. Clin. Med. $41,486-492$.

5. Grady, H. J. \& Lamar, M. A. (1952), Clin. Chem. 5, 542-551

6.. Burstein, M. \& Samaille, J. (1960), Clin. Chim. Acta 5, 609609

7. NIh-Manual of Lab. Operations (1974), Lipid. Res. Prog. 1.

8. Barr, D. P. (1951), Am. J. Med. 11, 480-493.

9. Havel, R. J., Eder, H. A. \& Bragdon, J. H. (1955), J. Clin. Invest. 34, 1345-1353

10. Carlson, L. A. \& Ericsson, M. (1975), Atherosclerosis 21, 417-435.

11. Mjфs, O. D., Thelle, D. S., Forde, O. H. \& Vik-Mo, H. (1977), Acta Med. Scand. 201, 323-329.

12. Castelli, W. P., Cooper, G. R., Doyle, J. T., Garcia-Palmers, M., Gordon, T., Hames, C., Hulley, S. B., Kagan, A., Kuchmak, M., McGee \& D. Vicic, W. (1977), J. Chron. Dis. 30, $147-169$

13. Wood, P. D., Haskell, W., Klein, H., Lewis, S., Stern, M. P. \& Farguhar, J. W. (1976), Metabolism 25, 1249-1257

14. Albers, J. J., Cheung, M. W. \& Hazżard, W. R. (1978), Metabolism 27, 479-489

15. Gordon, T., Castelli, W. P., Hjortland, M. C., Kannel, W. B. \& Dawber, T. R. (1977), Am. J. Med. 62, 707-714 zeigen, daß diese Beziehung bei Männern wahrscheinlich stärker als bei Frauen ausgeprägt ist. Vorbefunden entsprechend (14) kommen bei Männern mit gleicher VLDL-Triglyceridkonzentration zwar auch niedrigere HDL-Cholesterinkonzentrationen vor, im Gesamtspektrum des UZ-Lipidstatus, d.h. unter Einfluß eines differenzierten Lipidspektrums ist jedoch eine Abhängigkeit von VLDL-Triglyceriden bzw. VLDL-Cholesterin nicht auszuschließen.

In Zukunft wird es von Interesse sein, bei der Beurteilung des Risikofaktors erhöhter Cholesterinkonzentrationen in bestimmten Lipoproteinfraktionen auch den Einfluß endogener Triglyceridkonzentrationen zu berücksichtigen. Untersuchungsbefunde des UZ-Lipidstatus könnten bei endogenen Hypertriglyceridämien zur Arterioskleroseprophylaxe ebenfalls diagnostisch bedeutsam sein.

\section{Danksagung}

Frau M. E. Heggen möchten wir für die medizinisch-technische Durchführung der Arbeit herzlich danken.
16. Hulley, S. B., Cohen, R. \& Widdiwson, G. (1977), J. Am. Med. Assoc. 238, 2269-2271.

17. Logan, R. L., Thomson, M., Riemersma, R. A., Oliver, M. F., Olsson, A. G., Rössner, S., Callmer, E., Walldius, G., Kaijser, L., Carlson, L. A., Lockerbie, L. \& Lutz, H. (1978) Lancet $I$, 949-954

18. Furman, R. H., Alaupovic, P., Howard, R. P. (1967), in: Progress in Biochemistry and Pharmacology (D. Kritchvsky, R. Paoletti \& D. Steinberg, eds.), Vol. 2, Karger, Basel, S. 215.

19. Anderson, D. W., Nichols, A. V., Pan, S. S. \& Lindgren, F. T. (1978), Atherosclerosis 29, 161-179

20. Albers, J. J., Wahl, P. W., Cabana, V. G., Hazzard, W. R. \& Hoover, J. J. (1976), Metabolism 25, 633-644

21. Krauss, R. M., Lindgren, F. T., Silvers, A., Jutagir, R. \& Bradley, D. (1977), Clin. Chim. Acta 80, 465-470

22. Arntzenius, A. C., van Gent, C. M., van der Voort, H. \& Stegerhoek, C. I. (1978), Lancet $I, 1221$.

23. Borenstein, R., Stahl, N. L. \& Greenblatt, R. B. (1972), J. Am. Geriatr. Soc. 20, 369-376.

24. Carlson, L. A. \& Hardell, L. I. (1977), Eur. J. Clin. Invest. 7 , $133-135$

25. Glueck, Ch. J., Fallat, R. W., Millet, F. \& Steiner, P. M., (1975), Arch. Intern. Med. 135, 1025-1028.

26. Schäfer, E. J., Andersson, D. W., Brewer, J. B. jr., Levy, R. I., Danner, R. N. \& Blackwelder, W. C. (1978), Lancet II, 391-392
Priv. Doz. Dr. K. H. Vogelberg Diabetes-Forschungsinstitut Auf'm Hennekamp 65 D-4000 Düsseldorf 
